\title{
Pandemic advances alternatives to hallway medicine
}

\author{
n Cite as: CMAJ 2020 July 6;192:E789-90. doi: 10.1503/cmaj.1095880
}

Posted on cmajnews.com on June 19, 2020

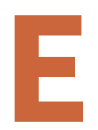
fforts to prevent the spread of coronavirus disease 2019 (COVID19) in Canadian hospitals have produced a surprising change in emergency departments: an abrupt end to hallway medicine. Hospital corridors are no longer overflowing with stretchers. Instead, social distancing in waiting areas, virtual emergency department visits, and a clear sight-line along every hallway have become the new normal.

Dr. Andrea Unger, chief of emergency services at Brant Community Health in south-west Ontario, says overcrowding had become the status quo in many emergency departments before the pandemic. "The rules on how many patients we managed in the emergency department were always different from other areas of the hospital," she says.

Delays in discharge, shortages of beds in hospitals and long-term care, and poor access to care in other parts of the health system all contributed to the seemingly intractable problem.

According to Unger, COVID-19 has been a wake-up call for hospital leaders. She insists her department cannot and will not return to pre-COVID levels of crowding. "Now, our overcrowding problem has become a risk to health care workers and the entire population, demanding more accountability; this is a fundamental shift," Unger says.

Emergency departments responded by setting up "hot" and "cold" zones, separating people who may have COVID19 from those without symptoms. Some converted classrooms and offices into extra examination rooms exclusively for patients with COVID-19.

Dr. Paul Hannam, chief of emergency medicine at North York General Hospital in

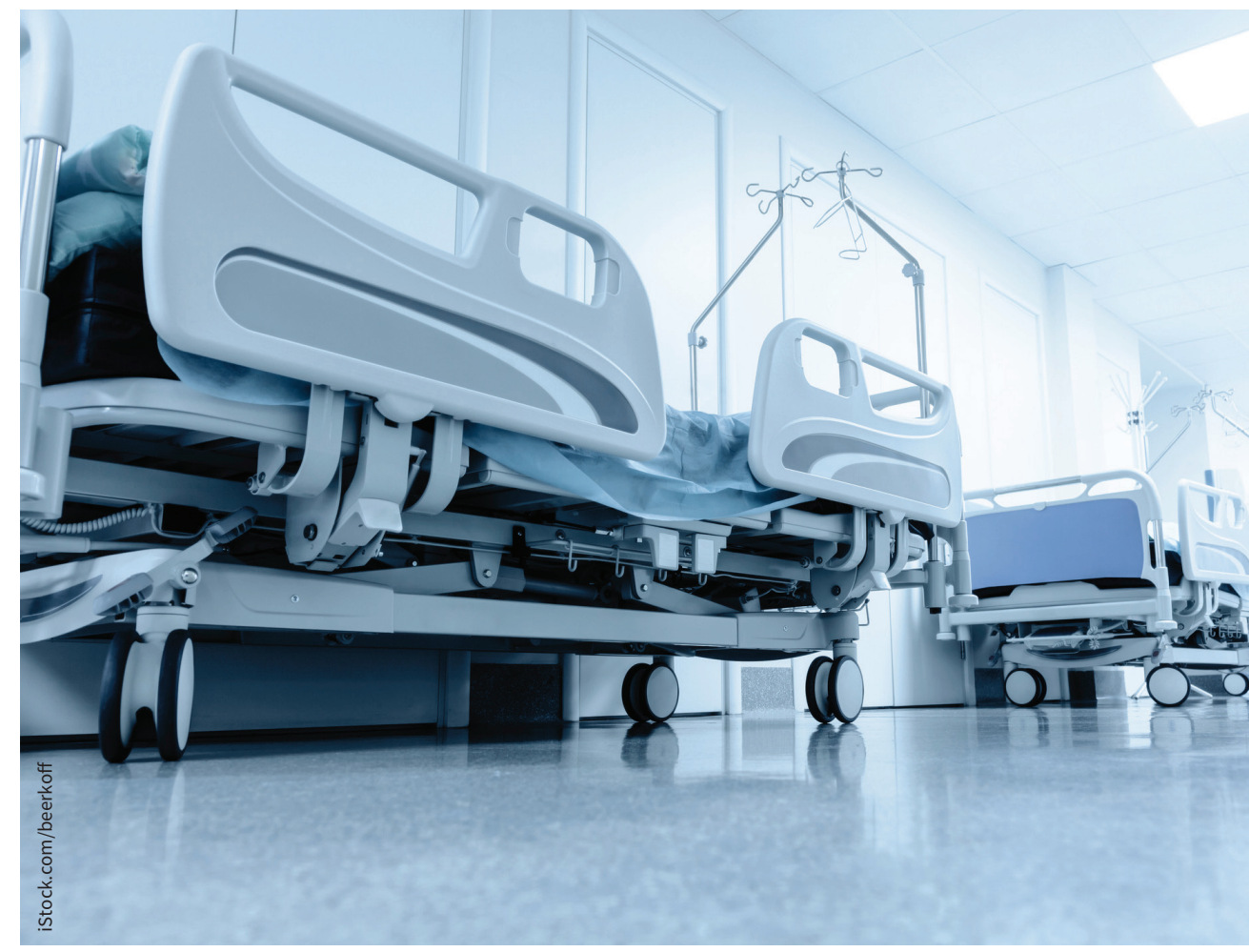

Emergency physicians say COVID-19 has forced creative solutions to overcrowding.

Toronto, says his department went a step further, setting up two separate hot zones - one for patients with mild symptoms and one for more severe cases.

While sicker patients are treated in an isolated area inside the hospital, "patients with mild symptoms never actually come inside the emergency department," Hannam explains. Instead, the hospital converted an ambulance driveway with a high roof into a "mini-field hospital" with eight beds. "We now even do chest $x$-rays outside," he says.

Beyond setting up temporary spaces to ease crowding, emergency departments are also finding ways to deliver care virtually.

William Osler Health System in Bramp- ton was already grappling with emergency department gridlock due to an influx of seasonal flu cases when COVID-19 emerged in Canada. According to emergency physician Dr. Prashant Phalpher, his community would be vulnerable if cases of COVID-19 surged because it has just one hospital bed per 1000 patients, below the Canadian average of 1.6.

So Phalpher and his team accelerated plans to deliver more care remotely. "Virtual care in emergency medicine would have been met with a lot of resistance a year ago," Phalpher says. "The pandemic made us ask, "Why not now?"

At Brampton Civic Hospital, patients who might have stayed a few days in the 
hospital with kidney or skin infections, asthma complications or COVID-19, now have the option to stay home, supported by a virtual health care team. An emergency doctor and nurse practitioner check in daily with these patients by phone and video chat and coordinate in-home nursing, radiology and specialist appointments as needed.

"Daily follow-up ensures the patient is doing well. If their condition changes, we can get them back into the hospital, avoiding the emergency department," Phalpher says.

Dr. Eddy Lang, head of emergency medicine at the Cummings School of Medicine in Calgary, believes the virtual care options adopted during the pandemic are here to stay, and Canadians may use emergency departments differently in the future. Lang says that broader uptake of virtual care in family medicine and other specialties may also reduce pressure on acute care as patients can access help elsewhere. "Emergency departments may not need to be as much of a safety net," he says.

In Alberta, the expansion of a callcentre service coordinating contact between long-term care homes and emergency departments was critical to keeping vulnerable patients out of the hospital, Lang adds.

Known as RAAPID, community doctors can call the service for advice when their patients need a higher level of care than they can provide. The service offers information on where beds are available and sends palliative care, and nursing supports to long-term care homes as required. In many cases, a single call may prevent unnecessary hospitalization, says Lang. He credits RAAPID with reducing the spread of COVID-19 between institutions and says the expanded service will continue for the foreseeable future.

As provinces reopen and patient volumes return to normal, Hannam says emergency departments must be vigilant and flexible to prevent a return to hallway medicine. "We know what crowding feels like with too many inpatients, sick patients in hallways, a full waiting room, and how uncomfortable that is. I can only hope the pandemic reinforces that crowding makes all of us vulnerable," he says.

Dr. Catherine Varner, Toronto, Ont. 